\title{
Unsubstituted Bicyclo[1.1.0]but-2-ylcarbinyl Cations
}

\author{
T. William Bentley, Bernd Engels, Thomas Hupp, Elena Bogdan, and Manfred Christl
}

\section{Supporting Information}

\section{Table of Contents}

Complete Reference (23).

Table 1. Absolute energies, sums of corrections to $\Delta G(298.15 \mathrm{~K})$,

and absolute energies for solvent environment.

Table 2. Cartesian coordinates of the structure $\mathbf{1 3}, \varepsilon=1$

Table 3. Cartesian coordinates of the structure $\mathbf{1 3}, \varepsilon=30$

Table 4. Cartesian coordinates of the structure endo-18(TS), $\varepsilon=1$

Table 5. Cartesian coordinates of the structure endo-18(TS), $\varepsilon=30$

Table 6. Cartesian coordinates of the structure endo-18'(TS), $\varepsilon=1$

Table 7. Cartesian coordinates of the structure endo-18'(TS), $\varepsilon=30$. S6

Table 8. Cartesian coordinates of the structure exo-18, $\varepsilon=1$ S6

Table 9. Cartesian coordinates of the structure exo-18, $\varepsilon=30$. S7

Table 10. Cartesian coordinates of the structure exo-18(TS), $\varepsilon=1$ S7

Table 11. Cartesian coordinates of the structure 25(TS), $\varepsilon=1$ S8 
Table 12. Cartesian coordinates of the structure 26, $\varepsilon=1$

Table 13. Cartesian coordinates of the structure $\mathbf{2 6}, \varepsilon=30$

S9

Table 14. Cartesian coordinates of the structure 27(TS), $\varepsilon=1$

$\mathrm{S} 10$

Table 15. Cartesian coordinates of the structure 27(TS), $\varepsilon=30$.

S10

Table 16. Cartesian coordinates of the structure 28, $\varepsilon=1$

S11

Table 17. Cartesian coordinates of the structure $\mathbf{2 8}, \varepsilon=30$

S12

${ }^{13} \mathrm{C}$ NMR spectra of the new compounds and the solvolysis products

S14-S27

\section{Complete Reference (23)}

Ahlrichs, R.; Bär, M.; Baron, H.-P.; Bauernschmidt, R.; Böcker, S.; Ehring, M.; Eichkorn, K.; Elliot, S.; Furche, F.; Haase, F.; Häser, M.; Horn, H.; Huber, C.; Huniar, U.; Kattaneck, M.; Kölmel, C.; Kollwitz, M.; May, K.; Ochsenfeld, C.; Öhm, H.; Schäfer, A.; Schneider, U.; Treutler, O.; Arnim, M. v.; Weigend, F.; Weis, P.; Weiss, H.; TURBOMOLE, Version 5.6; Universität Karlsruhe, Germany, since 1988.

Table 1. Absolute energies, sums of corrections to $\Delta G(298.15 \mathrm{~K})$, and absolute energies for solvent environment

\begin{tabular}{cccc}
\hline & B3LYP/TZVP & Corrections to $\Delta G$ & B3LYP/TZVP \\
& $\begin{array}{c}\text { (a.u.) } \\
\varepsilon=1\end{array}$ & $\varepsilon=1$ & (a.u.) \\
& & & $\varepsilon=30$ \\
\hline 13 & -194.327668 & 71.98831 & -194.419613 \\
endo-18(TS) & -194.263554 & 70.10102 & -194.353887
\end{tabular}




\begin{tabular}{cccc} 
endo-18'(TS) & -194.224770 & 70.41610 & -194.316520 \\
exo-18 & -194.263706 & 72.49391 & -194.353810 \\
exo-18(TS) & -194.233653 & 71.95329 & \\
25(TS) & -194.245765 & 69.20052 & \\
& & & \\
$\mathbf{2 6}$ & -194.321766 & 69.57780 & -194.413884 \\
& & & -272.969332 \\
$\mathbf{2 7 ( T S )}$ & -272.888392 & 104.06139 & -273.038954 \\
& & & \\
\hline $\mathbf{2 8}$ & -272.958013 & 105.21341 & \\
\hline
\end{tabular}

Table 2. Cartesian coordinates of the structure 13, $\varepsilon=1$

\begin{tabular}{|c|c|c|c|}
\hline \multicolumn{4}{|c|}{$E(B 3 L Y P / T Z V P)=-194.327668$} \\
\hline C & 0.494296 & 0.267751 & 1.238986 \\
\hline C & -0.896972 & 0.016059 & 0.695921 \\
\hline C & -0.897786 & 0.013269 & -0.693112 \\
\hline C & 0.491547 & 0.269770 & -1.240117 \\
\hline C & 0.756424 & -0.536774 & -0.001619 \\
\hline $\mathrm{H}$ & 0.865381 & 1.295861 & 1.203308 \\
\hline $\mathrm{H}$ & -1.683091 & -0.451683 & 1.289100 \\
\hline $\mathrm{H}$ & 0.918528 & -1.613147 & -0.003747 \\
\hline $\mathrm{H}$ & -1.684119 & -0.456757 & -1.284196 \\
\hline $\mathrm{H}$ & 0.672583 & -0.213872 & -2.200811 \\
\hline $\mathrm{H}$ & 0.859691 & 1.298830 & -1.202951 \\
\hline $\mathrm{H}$ & 0.676518 & -0.217605 & 2.198575 \\
\hline
\end{tabular}


Table 3. Cartesian coordinates of the structure 13, $\varepsilon=30$

\begin{tabular}{llll}
\multicolumn{4}{c}{ E (B3LYP/TZVP $)=-194.419613$} \\
C & 0.499380 & 0.266638 & 1.226393 \\
$\mathrm{C}$ & -0.876808 & 0.030852 & 0.689194 \\
$\mathrm{C}$ & -0.877276 & 0.031525 & -0.689299 \\
$\mathrm{C}$ & 0.499815 & 0.266142 & -1.226428 \\
$\mathrm{C}$ & 0.698461 & -0.560107 & 0.000168 \\
$\mathrm{H}$ & 0.902100 & 1.271417 & 1.158656 \\
$\mathrm{H}$ & -1.653286 & -0.452308 & 1.265854 \\
$\mathrm{H}$ & 0.834264 & -1.630377 & 0.000695 \\
$\mathrm{H}$ & -1.653817 & -0.450957 & -1.266381 \\
$\mathrm{H}$ & 0.670384 & -0.213802 & -2.180625 \\
$\mathrm{H}$ & 0.902692 & 1.270926 & -1.159500 \\
$\mathrm{H}$ & 0.670073 & -0.212551 & 2.180963
\end{tabular}

Table 4. Cartesian coordinates of the structure endo-18(TS), $\varepsilon=1$

$\begin{array}{lrrr}\text { E (B3LYP/TZVP })=-194.263554 & \\ \text { H } & -1.313204 & 1.543373 & 0.000000 \\ \text { H } & -2.738200 & 0.367846 & 0.000000 \\ \mathrm{C} & -1.662092 & 0.517842 & 0.000000 \\ \mathrm{C} & -0.815405 & -0.541903 & 0.000000 \\ \mathrm{C} & 1.208819 & 0.785983 & 0.000000 \\ \mathrm{H} & 0.703454 & 1.743647 & 0.000000 \\ \mathrm{H} & 2.291339 & 0.849218 & 0.000000 \\ \mathrm{H} & -1.193495 & -1.556051 & 0.000000 \\ \mathrm{C} & 0.656950 & -0.414873 & 0.696016 \\ \mathrm{C} & 0.656950 & -0.414873 & -0.696016\end{array}$




$\begin{array}{llll}\mathrm{H} & 0.855612 & -1.069912 & -1.532171 \\ \mathrm{H} & 0.855612 & -1.069912 & 1.532171\end{array}$

Table 5. Cartesian coordinates of the structure endo-18(TS), $\varepsilon=30$

$$
\begin{array}{cccc}
\text { E }(\mathrm{B} 3 \mathrm{LYP} / \mathrm{TZVP}) & -194.353887 & \\
\mathrm{H} & -1.299329 & 1.540947 & -0.000168 \\
\mathrm{H} & -2.722487 & 0.365371 & 0.003404 \\
\mathrm{C} & -1.649697 & 0.517858 & 0.000101 \\
\mathrm{C} & -0.805371 & -0.543884 & -0.000733 \\
\mathrm{C} & 1.198824 & 0.787120 & -0.001004 \\
\mathrm{H} & 0.684381 & 1.739328 & -0.002725 \\
\mathrm{H} & 2.278964 & 0.853290 & -0.002451 \\
\mathrm{H} & -1.186960 & -1.553410 & 0.002562 \\
\mathrm{C} & 0.650987 & -0.412959 & 0.697247 \\
\mathrm{C} & 0.648901 & -0.415131 & -0.695904 \\
\mathrm{H} & 0.863135 & -1.075418 & -1.520397 \\
\mathrm{H} & 0.862236 & -1.071769 & 1.523268
\end{array}
$$

Table 6. Cartesian coordinates of the structure endo-18'(TS), $\varepsilon=1$
$E(B 3 L Y P / T Z V P)=-194.224770$
$\begin{array}{llll}\text { C } & -1.607614 & 0.521130 & 0.000000\end{array}$
$\begin{array}{llll}\text { C } & -0.707578 & -0.606816 & 0.000000\end{array}$
$\begin{array}{llll}\text { C } & 0.543032 & -0.356969 & -0.787168\end{array}$
$\begin{array}{llll}\text { C } & 0.543032 & -0.356969 & 0.787168\end{array}$
$\begin{array}{llll}\text { C } & 1.204951 & 0.739889 & 0.000000\end{array}$ 

$\begin{array}{llll}\text { H } & 1.029487 & -1.073118 & 1.433581\end{array}$
$\begin{array}{llll}\text { H } & 1.029487 & -1.073118 & -1.433581\end{array}$
$\begin{array}{llll}\text { H } & -1.132270 & -1.612735 & 0.000000\end{array}$
$\begin{array}{llll}\mathrm{H} & -1.888662 & 1.011569 & -0.930222\end{array}$
$\begin{array}{llll}H & -1.888662 & 1.011569 & 0.930222\end{array}$

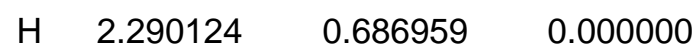
$\begin{array}{llll}H & 0.848589 & 1.760666 & 0.000000\end{array}$

Table 7. Cartesian coordinates of the structure endo-18' (TS), $\varepsilon=30$

\begin{tabular}{|c|c|c|c|}
\hline \multicolumn{4}{|c|}{$E(B 3 L Y P / T Z V P)=-194.316520$} \\
\hline C & -1.664741 & 0.014064 & -0.486372 \\
\hline C & -0.691370 & 0.005076 & 0.578271 \\
\hline C & 0.561306 & -0.782986 & 0.343973 \\
\hline C & 0.590087 & 0.755806 & 0.395656 \\
\hline C & 1.199419 & 0.012590 & -0.772905 \\
\hline $\mathrm{H}$ & 1.099294 & 1.375004 & 1.116271 \\
\hline $\mathrm{H}$ & 1.040680 & -1.474887 & 1.017309 \\
\hline $\mathrm{H}$ & -1.138257 & -0.028403 & 1.579056 \\
\hline $\mathrm{H}$ & -2.053300 & -0.914877 & -0.899308 \\
\hline $\mathrm{H}$ & -1.958663 & 0.943416 & -0.970038 \\
\hline H & 2.285358 & -0.008075 & -0.769484 \\
\hline $\mathrm{H}$ & 0.788047 & 0.053610 & -1.772369 \\
\hline
\end{tabular}

Table 8. Cartesian coordinates of the structure exo-18, $\varepsilon=1$
$E(B 3 L Y P / T Z V P)=-194.263706$
$\begin{array}{llll}\text { C } & -0.315196 & 1.674189 & 0.000000\end{array}$
$\begin{array}{llll}\text { C } & 0.396624 & 0.542125 & -0.694295\end{array}$ 


$\begin{array}{lrrr}\text { C } & 0.396624 & 0.542125 & 0.694295 \\ \text { C } & -0.495189 & -0.642192 & 0.000000 \\ \text { C } & 0.070228 & -1.877014 & 0.000000 \\ \text { H } & 1.148323 & -2.011876 & 0.000000 \\ \text { H } & -1.570081 & -0.510559 & 0.000000 \\ \text { H } & 1.058757 & 0.373012 & 1.529727 \\ \text { H } & 1.058757 & 0.373012 & -1.529727 \\ \text { H } & 0.196545 & 2.629820 & 0.000000 \\ \text { H } & -1.397505 & 1.719593 & 0.000000 \\ \text { H } & -0.542459 & -2.773668 & 0.000000\end{array}$

Table 9. Cartesian coordinates of the structure exo-18, $\varepsilon=30$

\begin{tabular}{|c|c|c|c|}
\hline \multicolumn{4}{|c|}{$E(B 3 L Y P / T Z V P)=-194.353810$} \\
\hline C & -0.317594 & 1.637857 & 0.000350 \\
\hline C & 0.379022 & 0.494427 & -0.694029 \\
\hline C & 0.379411 & 0.494346 & 0.695157 \\
\hline C & -0.505042 & -0.663567 & 0.003362 \\
\hline C & 0.070087 & -1.895623 & -0.001413 \\
\hline $\mathrm{H}$ & 1.146183 & -2.019988 & -0.006353 \\
\hline $\mathrm{H}$ & -1.583599 & -0.540144 & -0.004407 \\
\hline $\mathrm{H}$ & 1.043807 & 0.303806 & 1.524163 \\
\hline $\mathrm{H}$ & 1.039608 & 0.336038 & -1.530395 \\
\hline $\mathrm{H}$ & 0.210311 & 2.585202 & 0.003185 \\
\hline $\mathrm{H}$ & -1.400087 & 1.685678 & -0.001736 \\
\hline $\mathrm{H}$ & -0.540178 & -2.792344 & -0.004435 \\
\hline
\end{tabular}

Table 10. Cartesian coordinates of the structure exo-18(TS), $\varepsilon=1$

$E(B 3 L Y P / T Z V P)=-194.233653$ 


$\begin{array}{llll}\text { C } & 0.259663 & -2.006116 & -0.034621 \\ \text { C } & -0.065647 & -0.649193 & -0.092966 \\ \text { C } & 0.518064 & 0.534155 & -0.708298 \\ \text { C } & -0.384837 & 1.599857 & -0.136435 \\ \text { C } & 0.194991 & 0.586037 & 0.776145 \\ \text { H } & 0.819025 & -2.506408 & -0.821539 \\ \text { H } & -1.183578 & -0.819672 & -0.086408 \\ \text { H } & 0.785811 & 0.628575 & 1.677970 \\ \text { H } & 1.483358 & 0.621298 & -1.184132 \\ \text { H } & 0.051489 & 2.588608 & -0.045438 \\ \text { H } & -1.442082 & 1.597763 & -0.380794 \\ \text { H } & -0.171597 & -2.623189 & 0.748355\end{array}$

Table 11. Cartesian coordinates of the structure 25(TS), $\varepsilon=1$

$\begin{array}{lrrr}\text { E }(\mathrm{B} 3 \mathrm{LYP} / \mathrm{TZVP})= & -194.245765 & \\ \mathrm{C} & -0.075008 & -1.706228 & -0.011675 \\ \mathrm{C} & -0.651252 & -0.450998 & -0.086854 \\ \mathrm{C} & 1.104735 & -0.404541 & 0.796310 \\ \mathrm{C} & 0.409020 & 0.589036 & -0.025217 \\ \mathrm{C} & 0.505392 & 0.746112 & 1.510330 \\ \mathrm{H} & 0.772354 & -1.961982 & -0.640317 \\ \mathrm{H} & -1.658055 & -0.263178 & 0.286448 \\ \mathrm{H} & 2.084016 & -0.864967 & 0.829526 \\ \mathrm{H} & 0.748047 & 1.286327 & -0.776867 \\ \mathrm{H} & 1.240212 & 1.457113 & 1.878750 \\ \mathrm{H} & -0.380524 & 0.659924 & 2.125731 \\ \mathrm{H} & -0.606371 & -2.542561 & 0.432129\end{array}$


Table 12. Cartesian coordinates of the structure 26, $\varepsilon=1$

\begin{tabular}{|c|c|c|c|}
\hline \multicolumn{4}{|c|}{$E(B 3 L Y P / T Z V P)=-194.321766$} \\
\hline 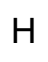 & 0.244196 & 2.583656 & 0.000000 \\
\hline & -0.297192 & 1.640855 & 0.000000 \\
\hline & -1.375940 & 1.761736 & 0.000000 \\
\hline & 0.199273 & 0.520040 & -0.880041 \\
\hline & 0.199273 & 0.520040 & 0.880041 \\
\hline & 0.880893 & 0.589881 & -1.724465 \\
\hline & 0.880893 & 0.589881 & 1.724465 \\
\hline & -1.226216 & -2.168837 & 0.000000 \\
\hline 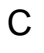 & 0.027331 & -0.545041 & 0.000000 \\
\hline 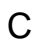 & -0.127285 & -2.004279 & 0.000000 \\
\hline & 0.289745 & -2.462328 & -0.896730 \\
\hline & 0.289745 & -2.4 & 0.896730 \\
\hline
\end{tabular}

Table 13. Cartesian coordinates of the structure 26, $\varepsilon=30$

\begin{tabular}{cccc}
\multicolumn{4}{c}{ E (B3LYP/TZVP $)=-194.413884$} \\
C & -0.081039 & -2.002301 & 0.000462 \\
C & -0.088263 & -0.539263 & -0.001409 \\
C & 0.188318 & 0.509288 & 0.861665 \\
C & 0.187602 & 0.511100 & -0.862742 \\
C & -0.249674 & 1.660906 & 0.001685 \\
H & 0.359641 & 2.557617 & 0.002250 \\
H & -1.316018 & 1.846852 & 0.001743 \\
H & 0.894331 & 0.531508 & -1.684096 \\
H & 0.897050 & 0.525567 & 1.681493 \\
H & -1.119309 & -2.345480 & -0.005146
\end{tabular}




$\begin{array}{llll}\mathrm{H} & 0.404591 & -2.392078 & -0.892420 \\ \mathrm{H} & 0.392770 & -2.389037 & 0.900206\end{array}$

Table 14. Cartesian coordinates of the structure 27(TS), $\varepsilon=1$

$\begin{array}{llll}\text { E }(\mathrm{B} 3 \mathrm{LYP} / \mathrm{TZV}) & -272.888392 & \\ \mathrm{C} & -0.012154 & 2.086523 & 0.000000 \\ \mathrm{C} & -0.739713 & 0.953781 & 0.000000 \\ \mathrm{C} & -0.037607 & -0.414858 & 0.693216 \\ \mathrm{C} & 0.814973 & -0.416359 & 1.907990 \\ \mathrm{C} & -0.037607 & -0.414858 & -0.693216 \\ \mathrm{C} & 0.814973 & -0.416359 & -1.907990 \\ \mathrm{C} & -0.987910 & -1.362416 & 0.000000 \\ \mathrm{H} & 1.072581 & 2.078726 & 0.000000 \\ \mathrm{H} & -1.822852 & 0.973124 & 0.000000 \\ \mathrm{H} & -0.688060 & -2.405757 & 0.000000 \\ \mathrm{H} & -2.057049 & -1.185668 & 0.000000 \\ \mathrm{H} & -0.494525 & 3.058522 & 0.000000 \\ \mathrm{H} & 1.656171 & 0.272698 & 1.836641 \\ \mathrm{H} & 0.230287 & -0.193258 & 2.800761 \\ \mathrm{H} & 1.211017 & -1.430996 & 2.018897 \\ \mathrm{H} & 1.656171 & 0.272698 & -1.836641 \\ \mathrm{H} & 1.211017 & -1.430996 & -2.018897 \\ \mathrm{H} & 0.230287 & -0.193258 & -2.800761\end{array}$

Table 15. Cartesian coordinates of the structure 27(TS), $\varepsilon=30$

$E(B 3 L Y P / T Z V P)=-272.969332$ 


$\begin{array}{llll}\mathrm{C} & -0.015823 & 2.070500 & -0.021664 \\ \mathrm{C} & -0.749560 & 0.941104 & -0.009416 \\ \mathrm{C} & -0.045109 & -0.401831 & 0.697296 \\ \mathrm{C} & 0.822862 & -0.389083 & 1.900149 \\ \mathrm{C} & -0.045078 & -0.415914 & -0.688822 \\ \mathrm{C} & 0.822544 & -0.425368 & -1.892057 \\ \mathrm{C} & -0.980793 & -1.366454 & 0.014098 \\ \mathrm{H} & 1.067069 & 2.056690 & -0.021792 \\ \mathrm{H} & -1.829614 & 0.960159 & -0.009653 \\ \mathrm{H} & -0.665131 & -2.402150 & 0.026673 \\ \mathrm{H} & -2.049242 & -1.195501 & 0.013238 \\ \mathrm{H} & -0.497132 & 3.039847 & -0.031462 \\ \mathrm{H} & 1.650790 & 0.310154 & 1.804218 \\ \mathrm{H} & 0.246061 & -0.156806 & 2.794306 \\ \mathrm{H} & 1.228305 & -1.397660 & 2.011378 \\ \mathrm{H} & 1.649926 & 0.276066 & -1.808539 \\ \mathrm{H} & 1.228872 & -1.435388 & -1.983825 \\ \mathrm{H} & 0.245570 & -0.209786 & -2.789570\end{array}$

Table 16. Cartesian coordinates of the structure 28, $\varepsilon=1$
$E(B 3 L Y P / T Z V P)=-272.958013$
$\begin{array}{llll}\text { C } & 0.962035 & 0.205795 & -0.197453\end{array}$
$\begin{array}{llll}\text { C } & 1.096215 & 0.190443 & 1.275133\end{array}$
$\begin{array}{llll}\text { C } & -0.278634 & -0.443340 & 1.143992\end{array}$
$\begin{array}{llll}\text { C } & -1.011389 & 0.105613 & 0.125426\end{array}$
C $\quad-0.146305 \quad 1.131580 \quad-0.587329$
$\begin{array}{llll}\mathrm{H} & 1.115788 & 1.164468 & 1.762293\end{array}$
$\begin{array}{llll}\mathrm{H} & -0.537413 & -1.368093 & 1.646971\end{array}$ 


$\begin{array}{llll}\mathrm{C} & 1.692387 & -0.670356 & -1.132317 \\ \mathrm{C} & -2.298650 & -0.395261 & -0.413740 \\ \mathrm{H} & -0.353630 & 1.198190 & -1.652536 \\ \mathrm{H} & -0.106117 & 2.118186 & -0.125624 \\ \mathrm{H} & 1.843219 & -0.486981 & 1.678088 \\ \mathrm{H} & -3.016584 & 0.426946 & -0.480198 \\ \mathrm{H} & -2.166793 & -0.775292 & -1.432235 \\ \mathrm{H} & -2.712627 & -1.191037 & 0.205051 \\ \mathrm{H} & 1.109309 & -0.922561 & -2.018398 \\ \mathrm{H} & 2.554942 & -0.079312 & -1.475439 \\ \mathrm{H} & 2.083329 & -1.567768 & -0.654605\end{array}$

Table 17. Cartesian coordinates of the structure $\mathbf{2 8}, \varepsilon=30$

\begin{tabular}{|c|c|c|c|}
\hline \multicolumn{4}{|c|}{$E(B 3 L Y P / T Z V P)=-273.038954$} \\
\hline C & 0.958334 & 0.206183 & -0.199195 \\
\hline C & 1.099874 & 0.186581 & 1.269936 \\
\hline C & -0.275256 & -0.444273 & 1.144720 \\
\hline C & -1.006503 & 0.108527 & 0.130927 \\
\hline C & -0.145468 & 1.134437 & -0.582413 \\
\hline $\mathrm{H}$ & 1.122758 & 1.160600 & 1.751350 \\
\hline $\mathrm{H}$ & -0.529119 & -1.371178 & 1.640929 \\
\hline C & 1.675375 & -0.671583 & -1.137492 \\
\hline C & -2.290254 & -0.394694 & -0.411821 \\
\hline $\mathrm{H}$ & -0.355278 & 1.200746 & -1.644489 \\
\hline $\mathrm{H}$ & -0.098891 & 2.116041 & -0.116364 \\
\hline $\mathrm{H}$ & 1.844370 & -0.494698 & 1.664417 \\
\hline $\mathrm{H}$ & -3.004625 & 0.427479 & -0.484211 \\
\hline
\end{tabular}




$\begin{array}{llll}\mathrm{H} & -2.146989 & -0.778736 & -1.424988 \\ \mathrm{H} & -2.700274 & -1.186946 & 0.211530 \\ \mathrm{H} & 1.079953 & -0.919540 & -2.014275 \\ \mathrm{H} & 2.532244 & -0.078883 & -1.483854 \\ \mathrm{H} & 2.063968 & -1.566524 & -0.657989\end{array}$




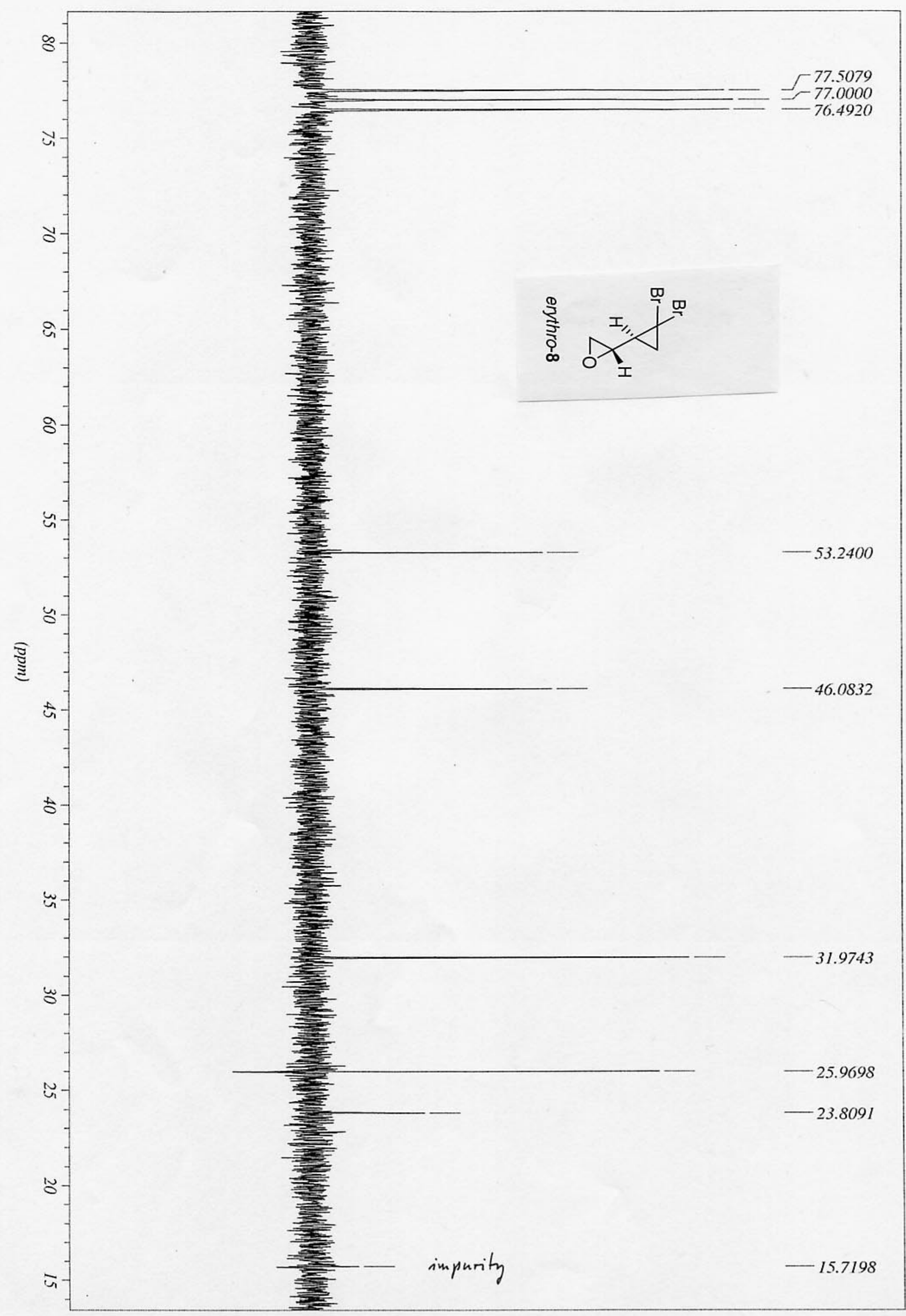




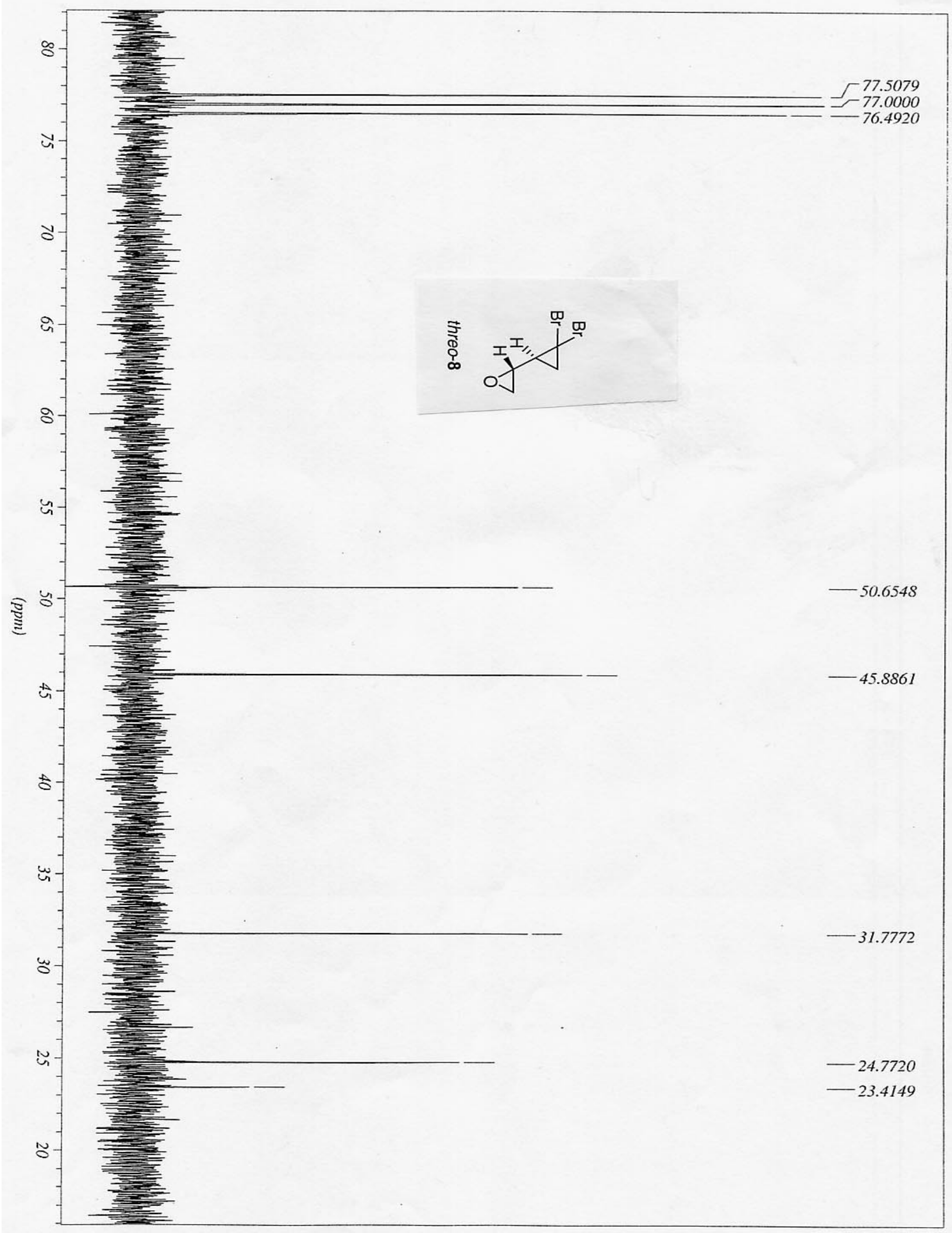



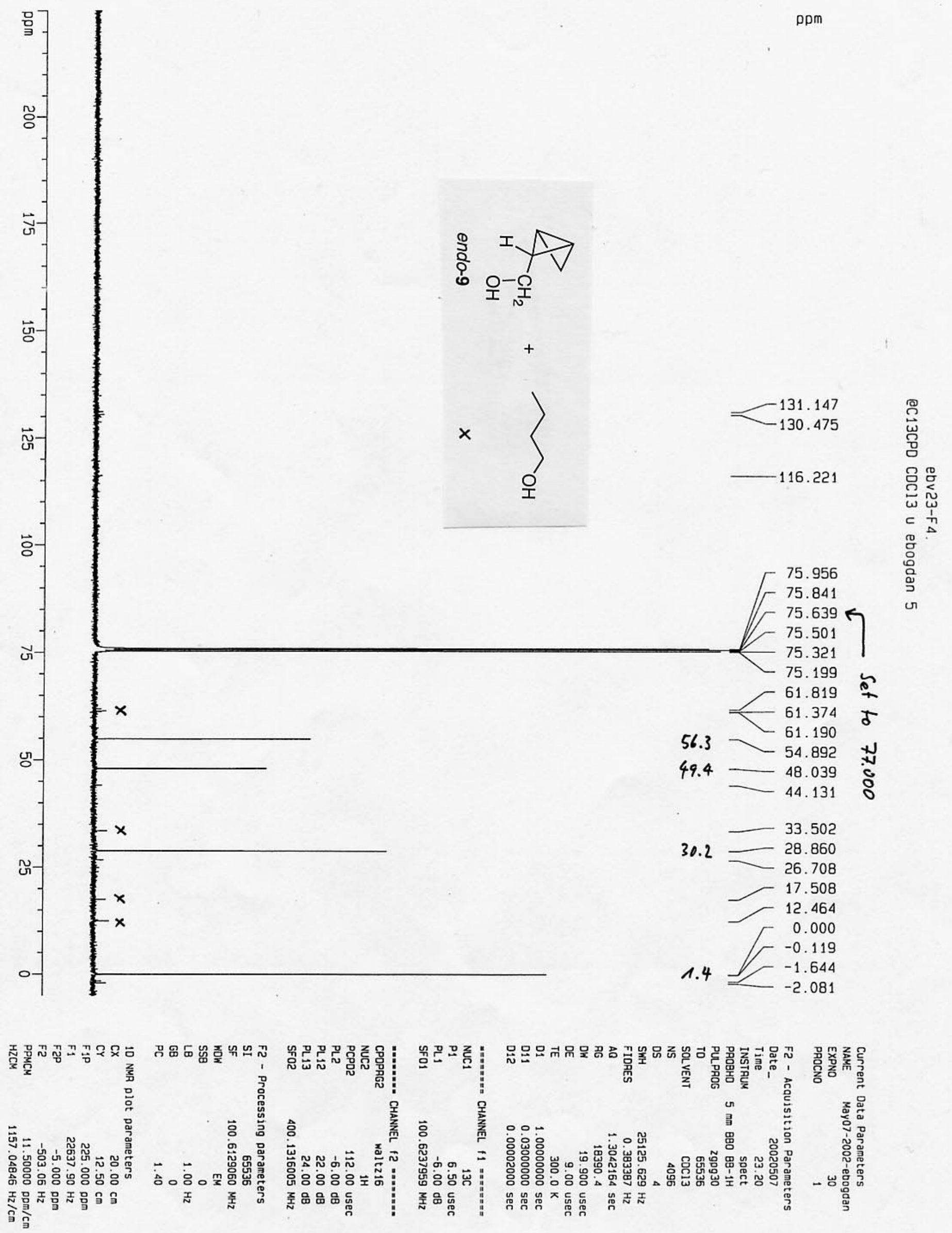

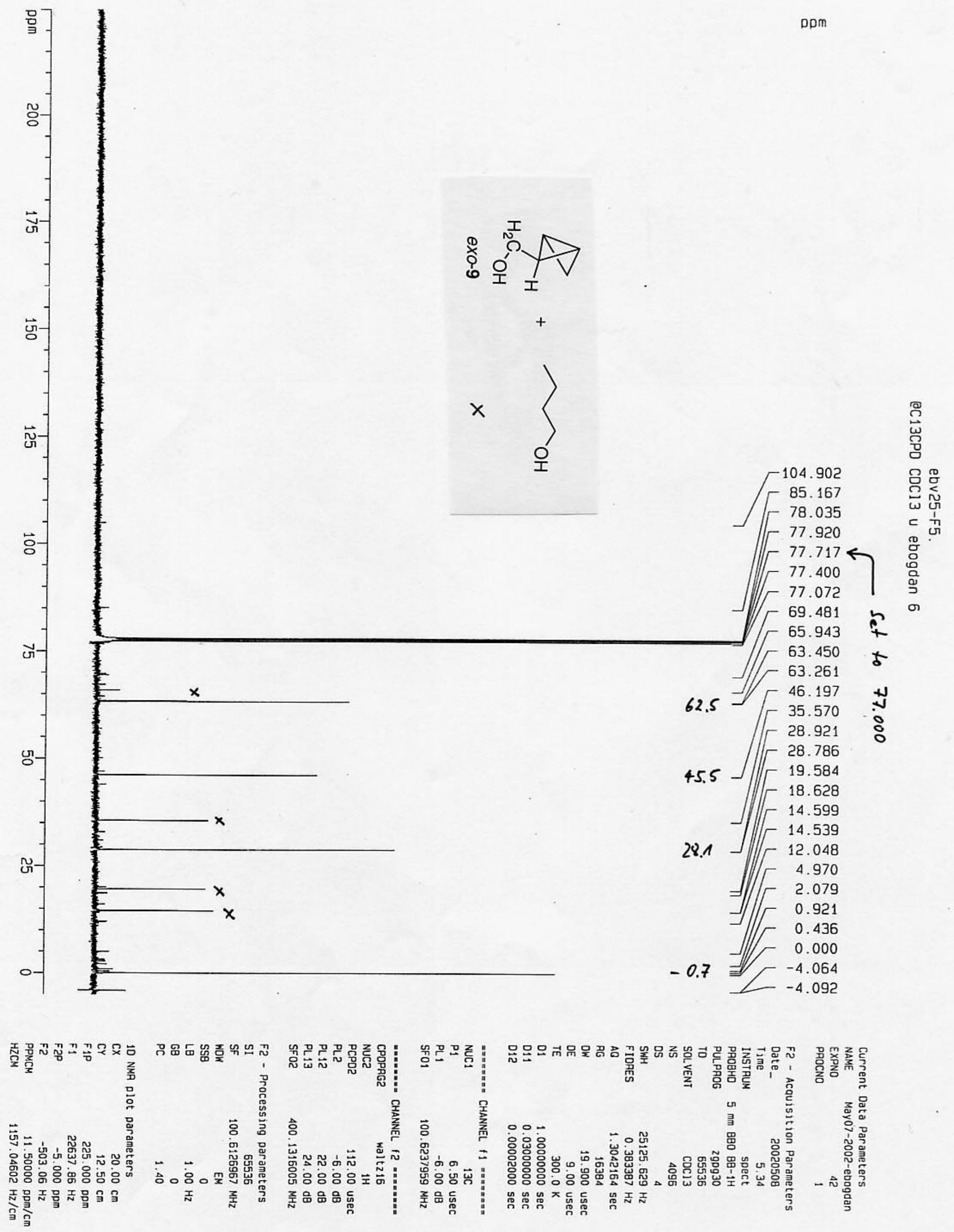

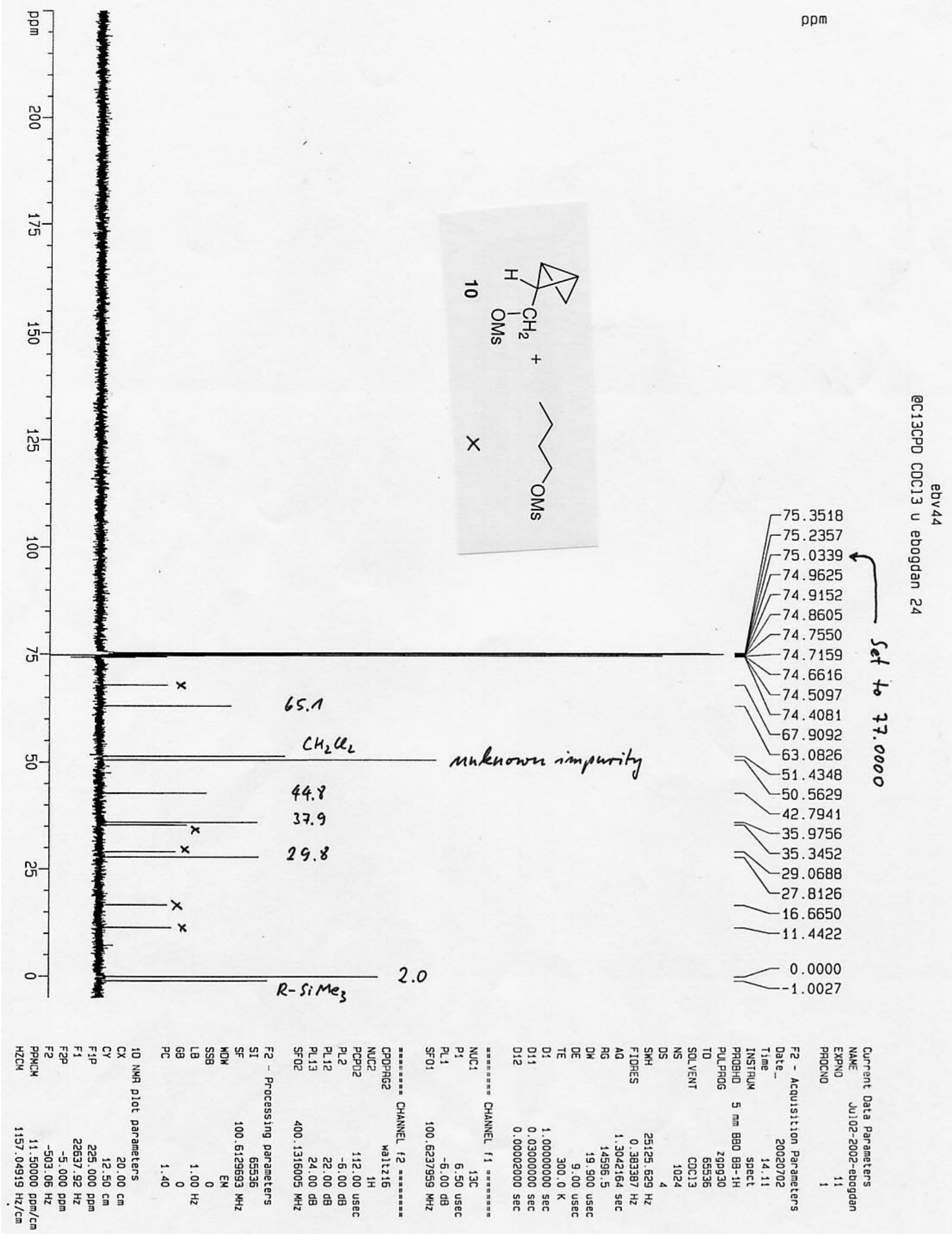


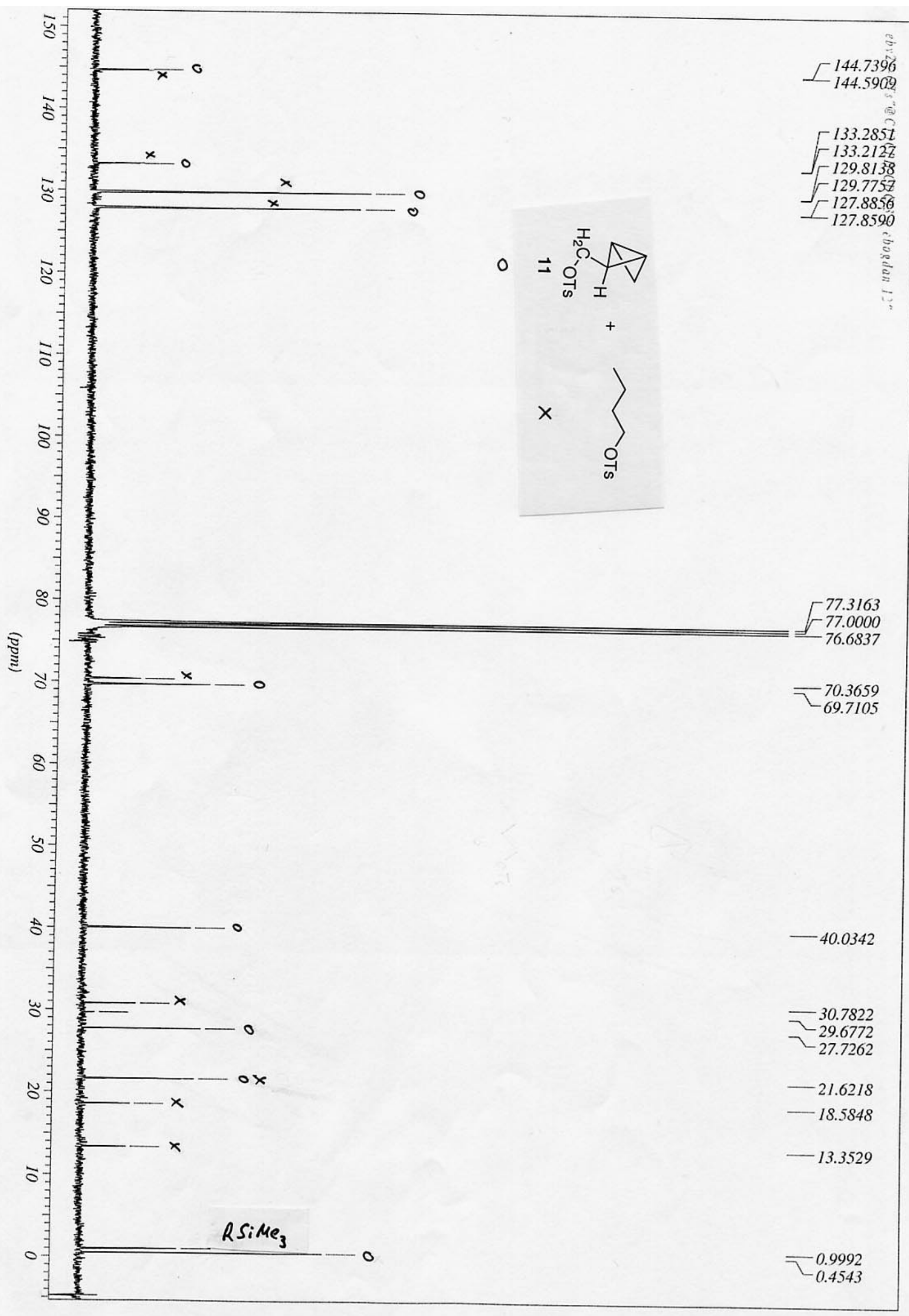




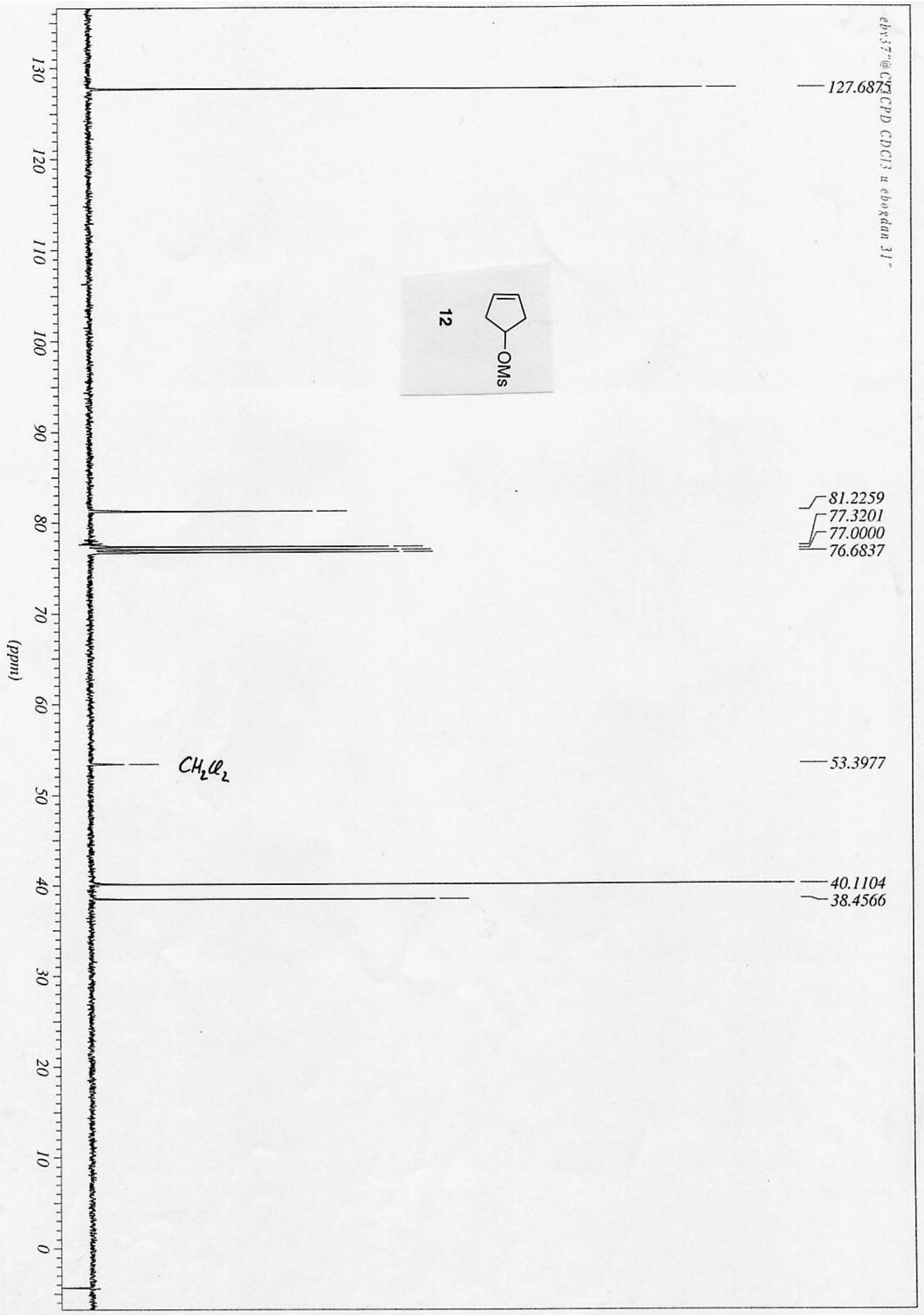




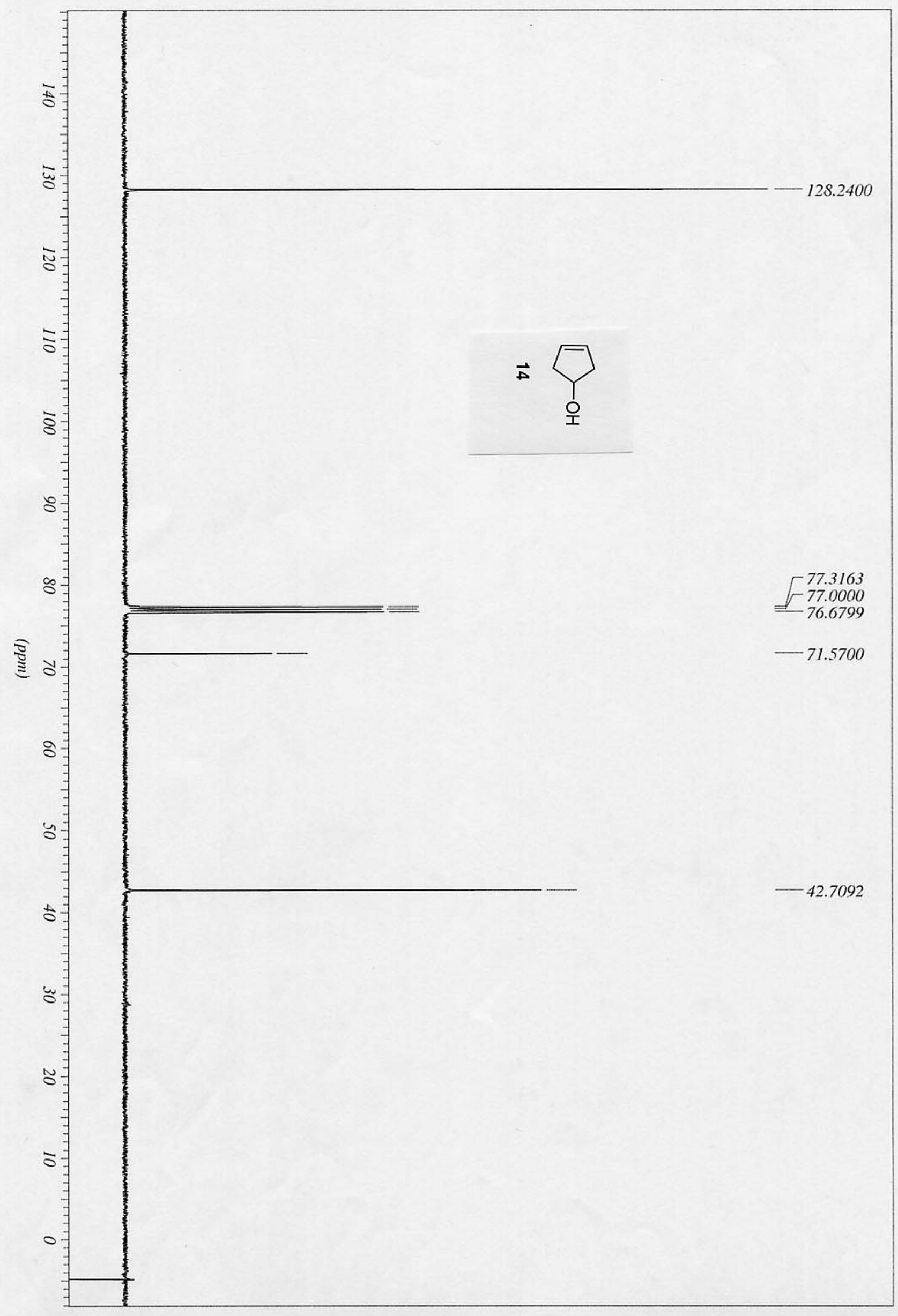

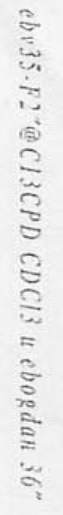









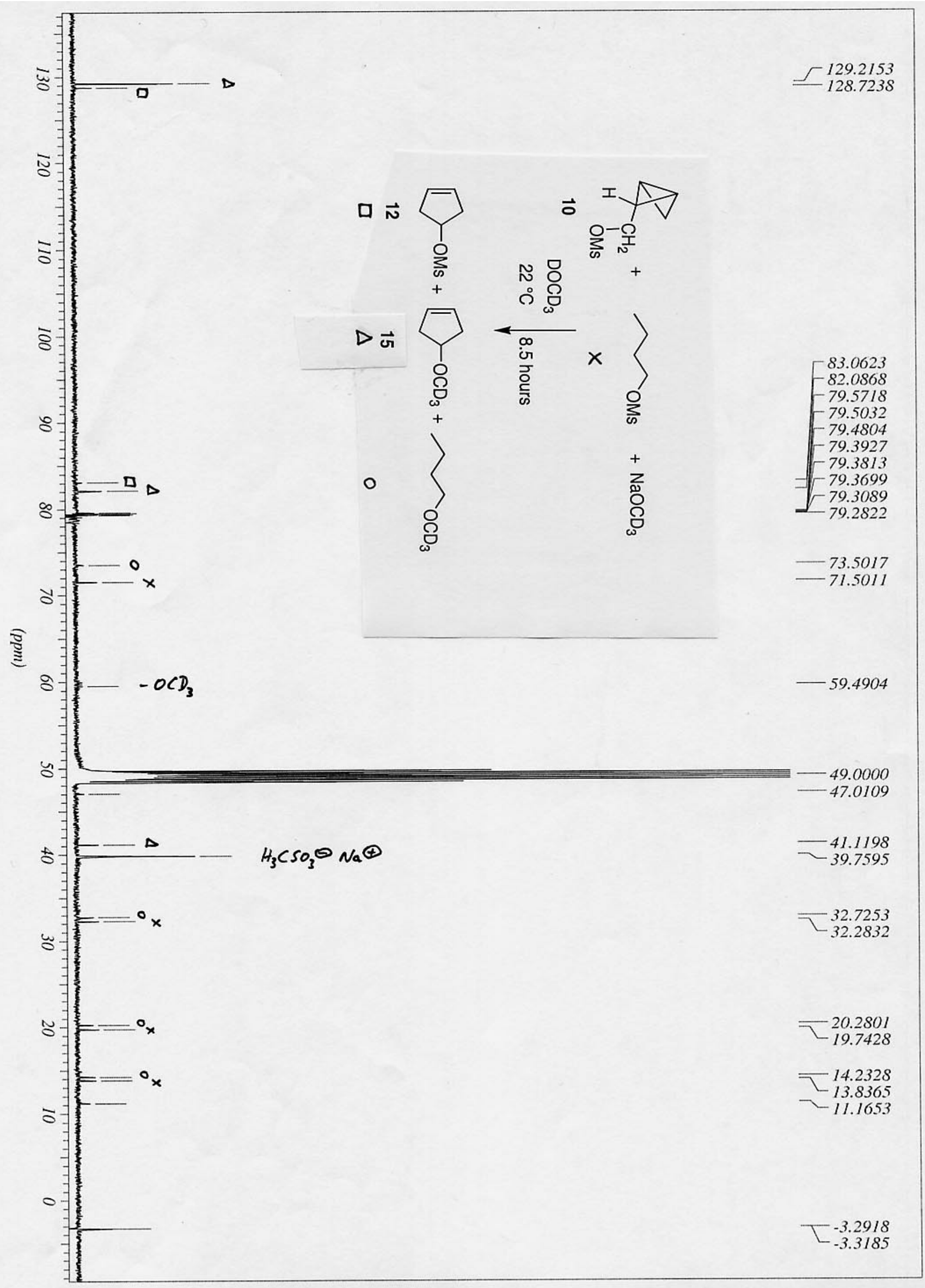




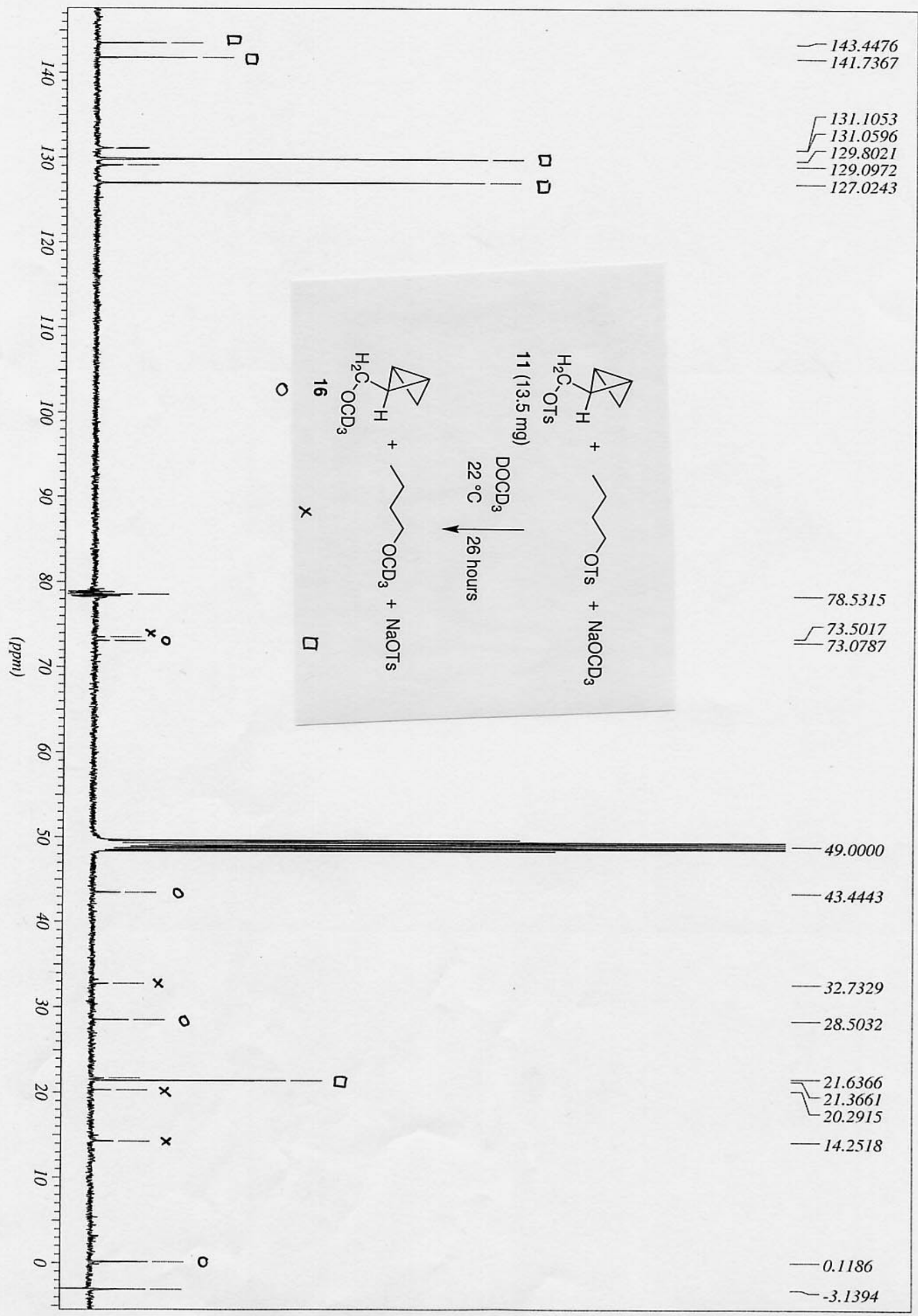



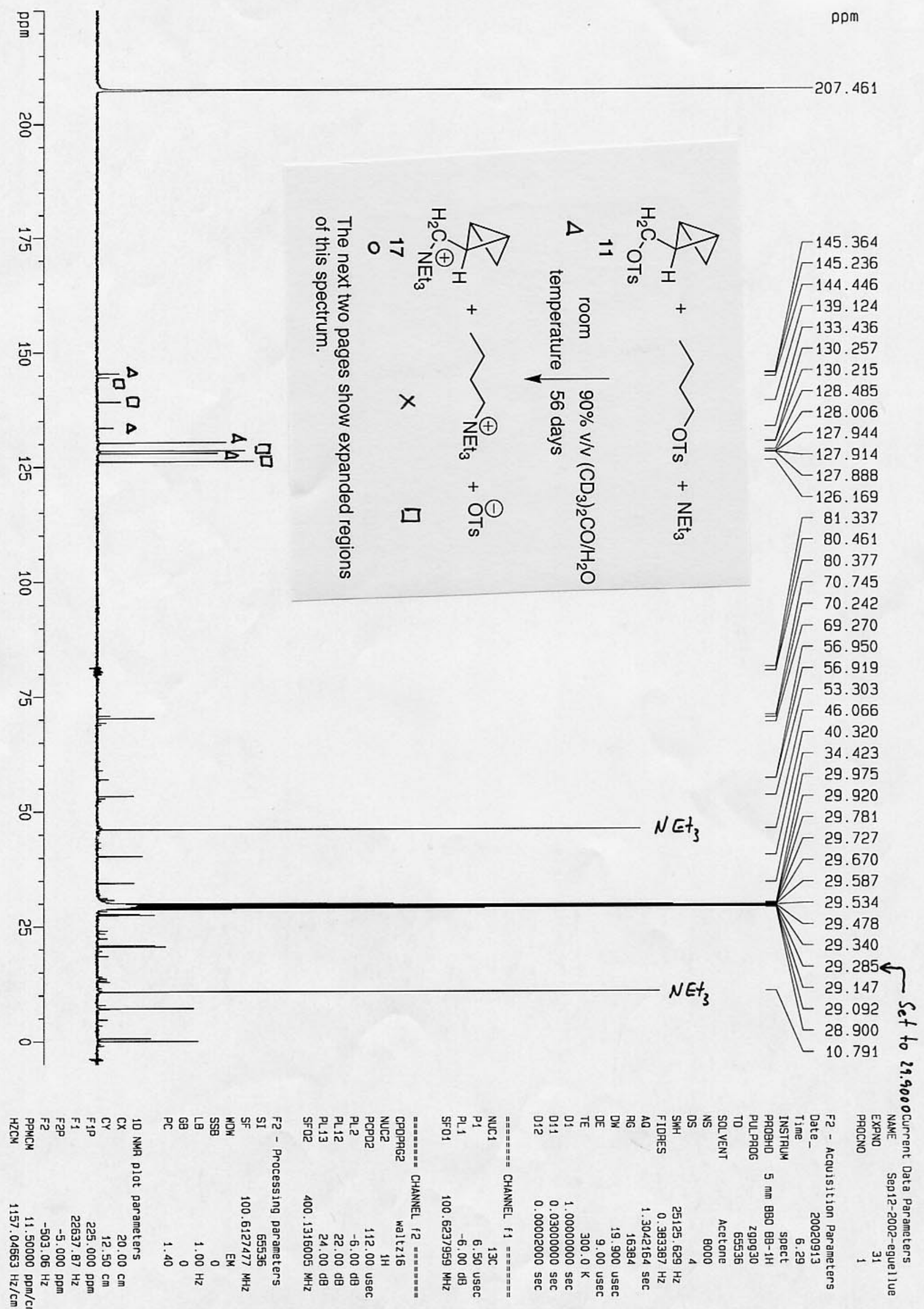


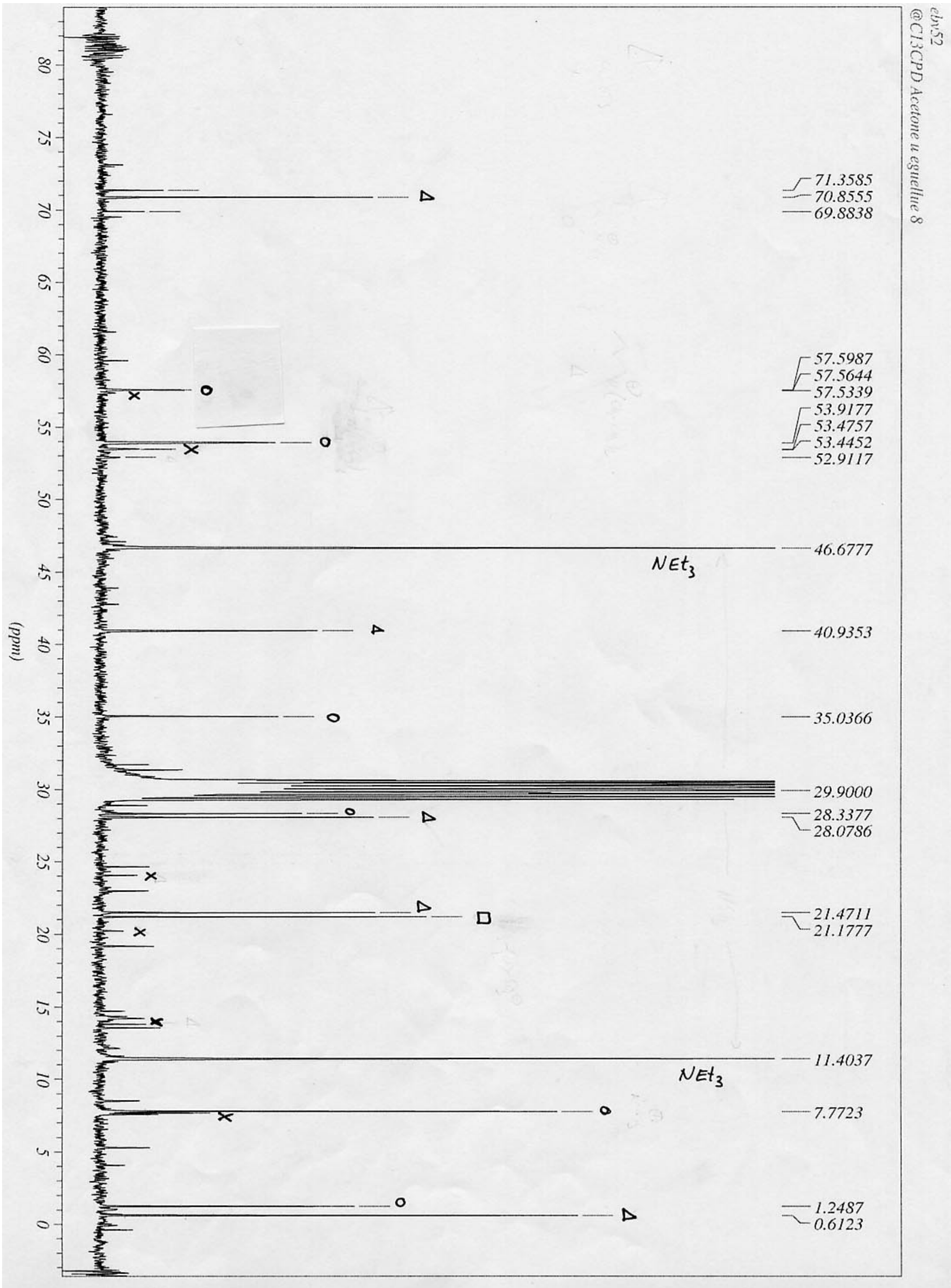




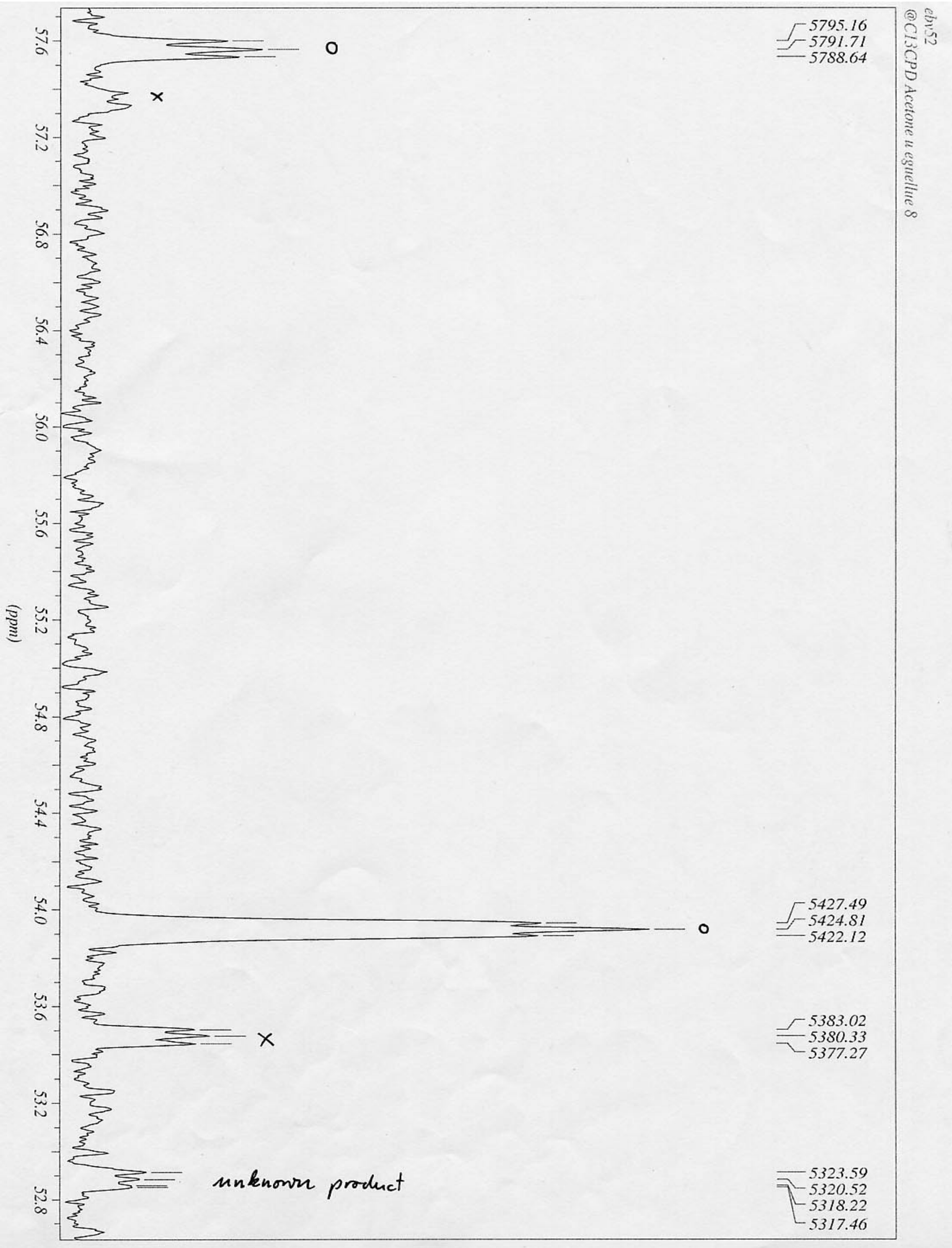

